\title{
The effects of selenium supplementation on antibody titres in patients with Hashimoto's thyroiditis
}

\author{
Lan-Feng Wang ${ }^{* 1,2}$, Rong-Xin Sun ${ }^{* 1,2}$, Chen-Fei Li $^{1,2}$, Xu-Hong Wang ${ }^{1,2}$ \\ ${ }^{1}$ Center for Endocrine Metabolism and Immune Diseases, Beijing Luhe Hospital, Capital Medical University, Beijing, China \\ ${ }^{2}$ Beijing Key Laboratory of Diabetes Research and Care, Beijing, China \\ *These authors contributed equally to this study
}

\begin{abstract}
Introduction: The objective of this study was to evaluate the effect of selenium supplementation on autoantibody titres, thyroid ultrasonography, and thyroid function in patients with Hashimoto's thyroiditis (autoimmune thyroiditis) and normal thyroid reference range. Material and methods: A total of 100 patients were given $200 \mathrm{ug} / \mathrm{d}$ selenium yeast orally, their thyroid function, levels of serum selenium, thyroid peroxidase antibodies (TPOAb), thyroglobulin antibodies (TGAb), and urine iodine were measured, and thyroid ultrasonography was performed before administration and three and six months afterwards, and the data were statistically analysed.

Results: The subjects exhibited a selenium deficiency before the administration of selenium, and the serum levels increased to moderate levels three and six months after the selenium supplementation $(p<0.05)$. The titres of TGAb decreased significantly in patients after six months of selenium supplementation $(\mathrm{p}<0.05)$. In the high antibody group, TgAb decreased after 6 months compared with baseline ( $p$ $=p<0.05)$, and TPOAb decreased after 3 and 6 months of selenium supplementation compared with baseline $(p<0.05)$.

Conclusion: In patients with autoimmune thyroiditis and normal thyroid reference range, there was a general selenium deficiency, but after six months of treatment it was shown that selenium supplementation may be effective in reducing the titres of TGAb and TPOAb. (Endokrynol Pol 2021; 72 (6): 666-667)
\end{abstract}

Key words: selenium yeast; Hashimoto's thyroiditis; thyroglobulin antibody; thyroid peroxidase antibody

\section{Introduction}

Due to the increasing prevalence of Hashimoto's thyroiditis [1-3], research concerning its prevention and control has been intensified. The results of different studies regarding the therapeutic effect of selenium supplementation on autoimmune thyroiditis have so far been inconsistent [4-5].

\section{Material and methods}

A total of 100 patients with autoimmune thyroiditis at the Endocrine Centre of Beijing Luhe Hospital affiliated to the Capital Medical University, who met the inclusion criteria, were enrolled in the study between August 2017 and October 2018. The patients were given 200 ug/d selenium yeast orally, and their thyroid function, levels of serum selenium, thyroid peroxidase antibodies (TPOAb), thyroglobulin antibodies (TGAb), and urine iodine were measured and thyroid ultrasonography was performed before administration and three and six months afterwards, and the data were statistically analysed. All the data were input into an Excel spreadsheet and imported into the SPSS 22.0 software for statistical analysis. The countable data were expressed as frequencies, and an $\chi^{2}$ test was used to compare the groups while measurement data with a normal distribution were expressed as $X \pm s$, and a paired samples t-test was used for the comparison. The non-normally distributed measurement data were expressed as medians (quartiles) (M [Q1, Q3]), and the Wilcoxon rank-sum test was used to make comparisons between the groups. $\mathrm{p}<0.05$ was considered statistically significant.

\section{Results}

The average initial serum selenium level was $72.93 \pm 43.242 \mathrm{ng} / \mathrm{mL}$, and the patients were in a selenium-deficient nutritional state, while the average initial urine iodine level was $110.80 \mathrm{ug} / \mathrm{L}$, which indicated that the patients were in an iodine-adequate nutritional state. After three and six months of selenium supplementation therapy, the overall serum selenium level in patients increased significantly $(p<0.05)$, while the urine iodine level remained relatively stable without any significant change ( $p>0.05$ in all) (Tab. 1).

The overall levels of TGAb were lower after three months of selenium supplementation therapy, but the difference was not statistically significant ( $p>0.05)$. After six months of selenium supplementation therapy, the overall levels of thyroid autoantibodies continued to decrease $(\mathrm{p}<0.05)$. For TPOAB, after 3 and 6 months of selenium supplementation, although there was a downward trend compared with baseline, there was no statistically significant difference ( $p \geq 0.05$ ).

On this basis, further stratified analysis was conducted. The baseline median antibody of all patients was taken as the cut-off point (TgAb 296.30U/mL, $\mathrm{TpoAb} 204.70 \mathrm{U} / \mathrm{mL}$ ), and patients with either of the two 
Table 1. The changes in thyroid function and the results of thyroid ultrasonography

\begin{tabular}{lccccc}
\hline Thyroid function & Initially & After $\mathbf{3}$ months & p-value & After $\mathbf{6}$ months & $\mathbf{p}$-value \\
\hline T3 $[\mathrm{ng} / \mathrm{mL}]$ & $1.10 \pm 0.154$ & $1.12 \pm 0.197$ & 0.082 & $1.10 \pm 0.198$ & 0.443 \\
\hline T4 $[\mathrm{ug} / \mathrm{dL}]$ & $7.20 \pm 1.51$ & $7.05 \pm 1.49$ & 0.327 & $7.30 \pm 1.69$ & 0.358 \\
\hline FT3 $[\mathrm{pg} / \mathrm{mL}]$ & $2.95 \pm 0.383$ & $3.02 \pm 0.347$ & 0.097 & $2.97 \pm 0.361$ & 0.540 \\
\hline $\mathrm{FT4}[\mathrm{ng} / \mathrm{dL}]$ & $1.35 \pm 1.35$ & $1.18 \pm 0.211$ & 0.251 & $1.21 \pm 0.242$ & 0.370 \\
\hline TSH $[\mathrm{ulU} / \mathrm{mL}]$ & $2.59[1.59,3.59]$ & $2.56[1.81,3.46]$ & 0.855 & $2.83[1.91,3.93]$ & 0.326 \\
\hline Thyroid ultrasonography $(\mathrm{n}=89)$ & & & & & 0.359 \\
\hline Diffuse compartmentalized or gridded changes & 61 & 57 & & & \\
\hline Diffuse gridded changes with a solitary thyroid nodule & 10 & 15 & & & \\
\hline Diffuse gridded changes with multiple thyroid nodules & 18 & 17 & & & \\
\hline
\end{tabular}

T3 — triiodothyronine; T4 — thyroxine; FT3 — free triiodothyronine; FT4 — free thyroxine; TSH — thyroid-stimulating hormone

antibodies higher than this cut-off point were divided into the high antibody group $(\mathrm{TgAb}>300 \mathrm{U} / \mathrm{mL}$ or $\mathrm{TpoAb}>200 \mathrm{U} / \mathrm{mL}$ ). Patients with both antibodies below this cut-off point were divided into the low antibody group $(\mathrm{TgAb}<300 \mathrm{U} / \mathrm{mL}$ and $\mathrm{TpoAb}<200 \mathrm{U} / \mathrm{mL})$. In the high-antibody group, TgAb decreased at 6 months from baseline $(p<0.05)$, but this difference was not significant at 3 months ( $p>0.05)$. TPOAb decreased at 3 and 6 months after Se supplementation compared with baseline (both $\mathrm{p}<0.05$ ). In the low antibody group, $\mathrm{TgAb}$ and $\mathrm{TpoAb}$ did not change significantly from baseline at either three or six months after Se supplementation ( $p>0.05)$ (Supplementary File - Tab. S1).

Effectiveness analysis was conducted for different groups of patients. A total of 100 patients were included in this study, with a loss of follow-up rate of $11 \%$, and 89 patients remained at the end of the study. There were 49 patients in the high antibody group and 40 patients in the low antibody group. In the above groups, the effective rate of patients in the high antibody group after supplementing selenium was significantly higher than that of the other two groups (Supplementary File - Tab. S2).

\section{Discussion}

At present, it may be related to insufficient sample size. Further stratified analysis showed that in patients with high antibody levels ( $\mathrm{TgAb}>300$ or TpoAb > 200), $\mathrm{TpoAb}$ antibodies decreased after three months of selenium supplementation, and both TpoAb and TgAb decreased after six months of selenium supplementation. However, no such effect was observed in the low antibody group. Since the antibody level reflects the activity of inflammation to some extent, this may be one of the main reasons for the better effect of selenium supplementation in people with high antibody levels, but this needs to be further confirmed by further stud- ies. In summary, selenium supplementation could be beneficial in the specific patient population with autoimmune thyroiditis described above.

Because the overall thyroid function in those patients remained relatively stable, the possibility that selenium supplementation might have a protective effect on the thyroid gland and stabilise thyroid function could not be excluded, and this needs to be verified in further studies.

\section{Conclusions}

Prevention of selenium deficiency may benefit some patients with autoimmune thyroiditis. However, before making the decision to supplement selenium, it would be advisable to adequately assess the overall status of the patient in order to obtain the most suitable intervention.

\section{Funding}

This study was funded by the Beijing Science and Technology Plan-Capital Characteristic: The effect of selenium supplementation on antibody titres of Hashimoto's thyroiditis under different iodine intake levels (No. Z171100001017071).

\section{References}

1. Caturegli P, De Remigis A, Rose NR. Hashimoto thyroiditis: clinical and diagnostic criteria. Autoimmun Rev. 2014; 13(4-5): 391-397, doi: 10.1016/j. autrev.2014.01.007, indexed in Pubmed: 24434360.

2. Vanderpump M. The epidemiology of thyroid disease. Br Med Bull. 2011; 99(1): 39-51, doi: 10.1093/bmb/ldr030, indexed in Pubmed: 21893493.

3. Rizzo M, Rossi RT, Bonaffini $\mathrm{O}$, et al. Increased annual frequency of Hashimoto's thyroiditis between years 1988 and 2007 at a cytological unit of Sicily. Ann Endocrinol (Paris). 2010; 71(6): 525-534, doi: 10.1016/j. ando.2010.06.006, indexed in Pubmed: 20817147.

4. Zhao H, Tian Y, Liu Z, et al. Correlation between iodine intake and thyroid disorders: a cross-sectional study from the South of China. Biol Trace Elem Res. 2014; 162(1-3): 87-94, doi: 10.1007/s12011-014-0102-9, indexed in Pubmed: 25161089.

5. Guastamacchia E, Giagulli VA, Licchelli B, et al. Selenium and Iodine in Autoimmune Thyroiditis. Endocr Metab Immune Disord Drug Targets. 2015; 15(4): 288-292, doi: 10.2174/1871530315666150619094242, indexed in Pubmed: 26088475. 